\title{
THE WORD AND ORDER PROBLEMS FOR SELF-SIMILAR AND AUTOMATA GROUPS
}

\author{
LAURENT BARTHOLDI AND IVAN MITROFANOV
}

\begin{abstract}
We prove that the word problem is undecidable in functionally recursive groups, and that the order problem is undecidable in automata groups, even under the assumption that they are contracting.
\end{abstract}

\section{INTRODUCTION}

Let $A$ be a finite set, and consider a group $G$ acting faithfully and "self-similarly" on the set $A^{*}$ of words over $A$. This means that every $g \in G$ acts in the form

$$
\left(a_{1} \ldots a_{n}\right)^{g}=a_{1}^{\prime}\left(a_{2} \ldots a_{n}\right)^{g^{\prime}}
$$

for some $a_{1}^{\prime} \in A$ and some $g^{\prime} \in G$ depending only on $a_{1}, g$; we encode them as $\left(g^{\prime}, a_{1}^{\prime}\right)=\bar{\Phi}\left(a_{1}, g\right)$ for a map $\bar{\Phi}: A \times G \rightarrow G \times A$. If furthermore $G$ is finitely generated (say by a finite set $S$, so $G$ is a quotient $F_{S} \rightarrow G$ of the free group on $S$ ), then its action may be described by finite data, namely a lift $\Phi: A \times S \rightarrow F_{S} \times A$ of the restriction of $\bar{\Phi}$ to the generators of $G$. A finitely generated group given in this manner is called functionally recursive [4, §3], or self-similar; we call $G$ the group presented by $\Phi$, and write $G=\langle\Phi\rangle$. We call $\Phi$ an (asynchronous) transducer.

Large classes of finitely generated groups can be presented as functionally recursive ones; notably, all the "iterated monodromy groups" of Nekrashevych [16], and the automata groups mentioned in $\$ 1.1$ below.

Even though the map $\Phi$ completely determines the action of $G$, and therefore $G$ itself, it is unclear how much of $G$ is known from $\Phi$. Our first result is as negative as can be:

Theorem A. There is no algorithm that, given $\Phi: A \times S \rightarrow F_{S} \times A$ and $s \in S$, determines whether $s=1$ in $\langle\Phi\rangle$.

1.1. Automata groups. Assume now that $G$ is a functionally recursive group, and that in the action (1.1) the elements $g^{\prime}$ have at most the length of $g$, in the generating set $S$. Then, up to replacing $S$ by $S \cup S^{-1} \cup\{1\}$, the map $\Phi$ takes the form $\Phi: A \times S \rightarrow S \times A$; we call it a finite state transducer. The group $G$ is called an automata group; these form a notorious class of groups, containing all finitely generated linear groups as well as infinite torsion groups such as the "Grigorchuk group" 7] and "Gupta-Sidki groups" [10. The Grigorchuk group is also a group of intermediate word-growth, and was used to settle the Milnor problem on group growth [8].

The action of $S$, and of $G$ itself, may be conveniently described by a finite labeled graph called its Moore diagram. Consider the directed graph $\Gamma$ with vertex set $S$

Date: October 25, 2017.

This work is supported by the "@raction" grant ANR-14-ACHN-0018-01. 


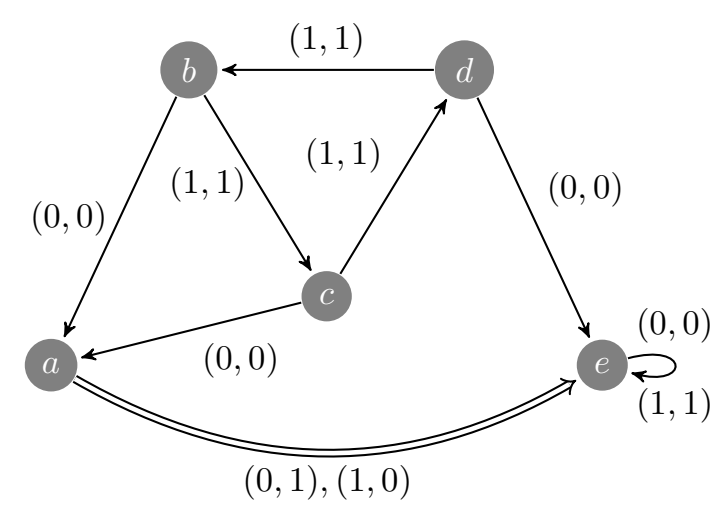

Figure 1. The transducer generating the Grigorchuk group. Here $A=\{0,1\}$ and $S=\{a, b, c, d, e\}$.

and an edge from $s$ to $t$ labeled $(a, b)$ whenever $\Phi(a, s)=(t, b)$; then the action of $s \in S$ on $A^{*}$ is determined as follows: given $a_{1} \ldots a_{n} \in A^{*}$, find the unique path in $\Gamma$ starting at $s$ and whose first label letters read $a_{1} \ldots a_{n}$; let $b_{1} \ldots b_{n}$ be the second label letters; then $\left(a_{1} \ldots a_{n}\right)^{s}=b_{1} \ldots b_{n}$. See Figure 1 for the graph $\Gamma$ describing the Grigorchuk group.

Every element of $G$ (say represented by a word $w$ of length $n$ in $S$ ) admits a similar description, but now using a graph with vertex set $S^{n}$. The word $w$ represents the identity in $G$ if and only if at every vertex reachable from $w$ all the outgoing edges have labels in $\{(a, a) \mid a \in A\}$. It follows that the word problem is decidable in $G$, and even belongs to LinSpace (and therefore to ExpTime); but that is about as much as is known. We consider the "order problem" (determine the order of an element), which was raised at the end of last century by Sidki and by Grigorchuk, Nekrashevych and Sushchansky [9, Problem 7.2.1(a)], to which Gillibert announced a solution in July 2017; his proof appears in [6]:

Theorem B. There is no algorithm that, given $\Phi: A \times S \rightarrow S \times A$ and $s \in S$, determines the order of $s$ in $\langle\Phi\rangle$, namely the cardinality of $\langle s\rangle$.

Worse than that, the action is uncomputable in the following sense: consider the natural extension of the action of $\langle\Phi\rangle$ to $A^{\infty}$. Then we have the following variants of Theorems $\mathrm{A}$ and $\mathrm{B}$

Theorem $\mathbf{A}^{\prime}$. There is no algorithm that, given $\Phi: A \times S \rightarrow F_{S} \times A$ and $a \in A$ and $s \in S$, determines whether $a^{\infty}$ is fixed by $s$.

Theorem B'. There is no algorithm that, given $\Phi: A \times S \rightarrow S \times A$ and $a \in A$ and $s \in S$, determines the cardinality of the orbit of $a^{\infty}$ under $\langle s\rangle$.

Finally, the results in Theorems $\mathrm{A}$ and $\mathrm{B}$ can be made uniform as follows:

Theorem A". There is a functionally recursive group $\langle\Phi\rangle$ with $\Phi: A \times S \rightarrow F_{S} \times A$ such that $\left\{s \in F_{S} \mid s=1\right.$ in $\left.\langle\Phi\rangle\right\}$ is not recursive.

Theorem B". There is an automata group $\langle\Phi\rangle$ with $\Phi: A \times S \rightarrow S \times A$, and two states $s, t \in S$, such that the set $\left\{n \in \mathbb{N} \mid s^{n}\right.$ has finite order $\}$ is not recursive. 
1.2. Contracting groups. Assume now that $G$ is a functionally recursive group, and that in the action (1.1) the elements $g^{\prime}$ are shorter than $g$, in the generating set $S$, in the sense that there are constants $\lambda<1$ and $C$ with $\left|g^{\prime}\right| \leqslant \lambda|g|+C$ for all $g \in G$. Then, up to replacing $S$ by the set of all words of length $\leqslant C /(1-\lambda)$, we also have $\left|g^{\prime}\right| \leqslant|g|$; we have thus defined a subclass of automata groups, called contracting automata groups (see 3.4 for a more precise definition). Their word problem is decidable in LogSpace (and therefore in PolyTime). We will see, however, that the order and orbit order problems remain unsolvable in that restricted class:

Theorem $\mathbf{C}$ (= Theorem 3.4). The transducers constructed in Theorems B and $B^{\prime}$ may be assumed to generate contracting groups.

1.3. Sketch of proofs. We encode Minsky machines in functionally recursive groups. Minsky machines (see [15]) are restricted Turing machines with two tapes, which may move the tapes and sense the tape's end but may not write on them; equivalently, they are finite state automata equipped with two counters with values in $\mathbb{N}$ that may be incremented, decremented and tested for 0 .

In all cases, we encode the machine, in state $s$ with counter values $(m, n)$, by the word $s x^{2^{m}} y^{2^{n}}$ in a functionally recursive group containing elements $x, y$ and an element $s$ for each state of the machine. The action of the group is so devised that if the machine evolves to state $\left(s^{\prime}, m^{\prime}, n^{\prime}\right)$ then the recursive action is given by $s^{\prime} x^{2^{m^{\prime}}} y^{2^{n^{\prime}}}$. The image of a prescribed ray under $s x^{2^{m}} y^{2^{n}}$ records the computational steps of the machine when started in $(s, m, n)$, and in particular whether the machine reached a final state. We construct an auxiliary element $t$ that only acts on sequences containing a trace of this final state, and then $\left(s x^{2^{m}} y^{2^{n}}\right) t\left(s x^{2^{m}} y^{2^{n}}\right)^{-1}$ fixes the original ray if and only if the machine never reaches the final state. Taking the commutator of that last element acting only in the neighbourhood of the original ray yields an expression that is trivial if and only if the machine never reaches the final state.

Inherently, sometimes the output of the transducer is longer than the input (e.g., if the machine increments the first counter, the transducer must replace $x$ by $x^{2}$ ). To obtain an automata group, we have the transducer consume a power of its input word $s x^{2^{m}} y^{2^{n}}$; e.g., the incrementation of the counter may be performed by erasing every second $s$ and every second block of $y^{2^{n}}$, s. The element $s x^{2^{m}} y^{2^{n}}$ then may be arranged to finite order if and only if the machine reaches the final state.

1.4. Tilings. Our results on functionally recursive groups and transducers may also be interpreted in terms of tilings. Let $C$ be a finite set of colours, and $T \subseteq C^{N, E, S, W}$ be a set of Wang tiles. A valid tiling is a map $t: \mathbb{Z}^{2} \rightarrow T$ with $t(x, y)^{N}=t(x, y+1)^{S}$ and $t(x, y)^{E}=t(x+1, y)^{W}$ for all $x, y \in \mathbb{Z}^{2}$. Berger showed in 2] that it is undecidable to determine, given $T$, whether there exists a valid tiling by $T$. This has been improved: for $\lambda, \mu \in\{N, E, S, W\}$, call a set of tiles $\lambda \mu$-deterministic if for every $c, d \in C$ there exists at most one tile $u \in T$ with $u^{\lambda}=c$ and $u^{\mu}=d$, and $\lambda \mu$-complete if there exists precisely one tile $u \in T$ with these conditions. Lukkarila showed in [14 that the undecidability result holds even under the restriction that $T$ is $N E, N W, S E, S W$-deterministic. Clearly a $S W$-complete tileset tiles uniquely the first quadrant for any choice of colours on the axes.

Our result on the order problem has the following translation into tilings. We consider tilings of the upper half-plane $\{(x, y) \mid y \geqslant 0\}$. Then the following problem is undecidable even for $S E, S W$-complete tilesets: "given $c \in C$, is there an integer 
$n \in \mathbb{N}$ such that every tiling of the upper half-plane with $c^{\infty}$ on the horizontal axis is horizontally n-periodic?".

Indeed, given $\Phi: A \times S \rightarrow S \times A$, set $C=A \sqcup S$ and whenever $\Phi(a, s)=$ $\left(s^{\prime}, a^{\prime}\right)$ build a tile with $N, E, S, W$-labels $s^{\prime}, a^{\prime}, s, a$ respectively; also build tiles with $N, E, S, W$-labels $c, d, c, d$ for all $(c, d) \in C^{2} \backslash(S \times A)$. Then the above tiling problem is satisfied for $c \in S$ if and only if $c$ has finite order in $\langle\Phi\rangle$.

The word problem may also be translated to a tiling problem, but now in hyperbolic space. The tileset is now $T \subseteq C^{N, E, S_{1}, S_{2}, W}$. The lattice $\mathbb{Z}^{2}$ is now $\Lambda:=\left\{2^{y}(i+\right.$ $x) \mid x, y \in \mathbb{Z}\} \subset \mathbb{H}$. A tiling is a map $t: \Lambda \rightarrow T$ with $t\left(2^{y}(i+x)\right)^{E}=t\left(2^{y}(i+x+1)\right)^{W}$ and $t\left(2^{y}(i+2 x)\right)^{N}=t\left(2^{y+1}(i+x)\right)^{S_{1}}$ and $t\left(2^{y}(i+2 x+1)\right)^{N}=t\left(2^{y+1}(i+x)\right)^{S_{2}}$ for all $x, y \in \mathbb{Z}$. Tiles are visualized as pentagons assembling into a tiling of the hyperbolic plane, invariant under the transformations $z \mapsto z+1$ and $z \mapsto 2 z$ :

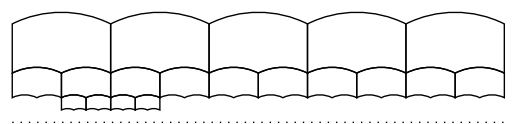

The following problem is undecidable even for $N E, N W$-complete tilesets: "given $c \in C$, does every tiling of $\{x+i y \in \mathbb{H} \mid x \in[0,1], y \leqslant 1\}$ with $c$ on the edge from $i$ to $i+1$ have identical labels on the boundary half-lines $\{x=0\}$ and $\{x=1\}$ ?".

Indeed by subdividing and inserting the empty state we may assume that the map $\Phi$ describing our functionally recursive group satisfies $\Phi(A \times S) \subseteq S^{2} \times A$; then tiles are defined as above.

1.5. History. Links have been established since the beginning between undecidable problems in theoretical computer science - halting of Turing machines and in algebra - decidability of properties of algebraic objects. Minsky machines, because of their simplicity, have been early recognized as useful tools in this correspondence, see e.g. Gurevich's work [11] on identities in semigroups.

Automata semigroups are defined quite similarly to automata groups; one merely drops the requirement that the action be by invertible maps. Decision problems have been extensively studied within the class of automata semigroups [1, 13. Gillibert proved in [5] that the order problem is unsolvable in that class. His proof is based on the undecidability of Wang's tiling problem [2, and harnesses Kari's solution of the nilpotency problem for cellular automata [12].

There are usually serious difficulties in converting a solution in semigroups to one in groups. In particular, the tilings at the heart of Gillibert's construction give fundamentally non-invertible transformations of $A^{*}$.

On the other hand, a direct approach to the order problem succeeded for the restricted class of "bounded automata" groups; Bondarenko, Sidki and Zapata prove in [3] that they have solvable order problem. Gillibert announced in [6] the undecidability of the order problem in automata groups; his work uses a simulation of arbitrary Turing machines by transducers via cellular automata.

\section{Functionally ReCURsive groups And Minsky machines}

All our theorems are proven by embedding Minsky machine computations into functionally recursive groups. Let us recall more precisely the definition of these machines:

Definition 2.1. A Minsky machine is a computational device $\mathbf{M}$ equipped with two integer counters $m, n$ and a finite amount of additional memory. It has a finite 
set $S$ of states, an initial state $s_{*} \in S$, a final state $s_{\dagger} \in S$, and for each state $s \neq s_{\dagger}$ an instruction, which can by any of the following kind:

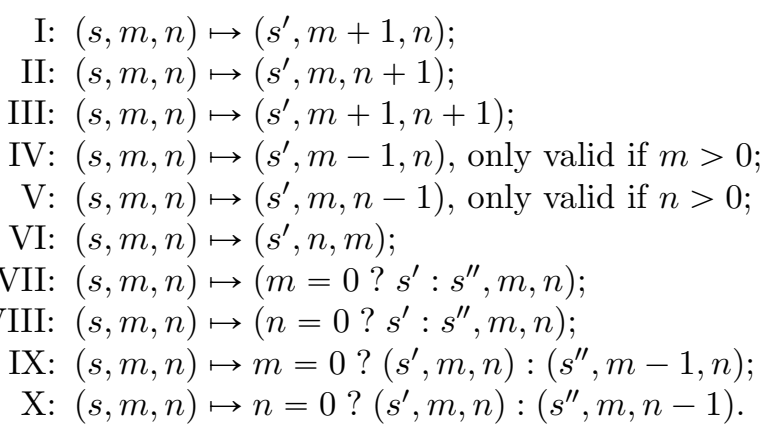

(We use the C style "?:" operator, with ' $a$ ? $b: c$ ' meaning 'if $a$ then $b$ else $c$ '.)

As $\mathbf{M}$ is turned on, its state and counters initialize at $\left(s_{0}, m_{0}, n_{0}\right)=\left(s_{*}, 0,0\right)$, and then $\left(s_{i+1}, m_{i+1}, n_{i+1}\right)$ is determined from $\left(s_{i}, m_{i}, n_{i}\right)$ using the prescribed rules. If at some moment $s_{i}=s_{\dagger}$ then $\mathbf{M}$ stops; otherwise it runs forever.

We recall the main result on Mealy machines, namely that they are as powerful as Turing machines:

Proposition 2.2 (15]). (1) There is no algorithm that, given a Minksy machine $\mathbf{M}$, determine whether it stops.

(2) There is a "universal" Minsky machine $\mathbf{M}$ such that

$$
\left\{n \in \mathbb{N} \mid \mathbf{M} \text { stops when turned on in state }\left(s_{*}, 0, n\right)\right\}
$$

is not recursive.

We also note that only one of the instructions $\{\mathrm{I}, \mathrm{II}\}$ and III is necessary, and that in the presence of VI only one of I,II, one of IV,V, one of VII,VIII and one of IX,X is necessary. Minimal sets of instructions are $\{\mathrm{III}, \mathrm{IV}, \mathrm{V}, \mathrm{VII}, \mathrm{VIII}\}$ and $\{\mathrm{I}, \mathrm{IV}, \mathrm{VI}, \mathrm{VII}\}$ and $\{$ III,IX,X $\}$ and $\{$ I,VI,IX $\}$.

2.1. Proof of Theorem $\mathbf{A}^{\prime}$. Let $\mathbf{M}$ be a Minsky machine with stateset $S_{0}$. Without loss of generality, we assume that all instructions of $\mathbf{M}$ are of type I, VI, IX.

We construct a functionally recursive group $\left\langle\Phi_{\mathbf{M}}\right\rangle$ presented by $\Phi_{\mathbf{M}}: A \times S \rightarrow$ $F_{S} \times A$, for sets $A, S$ given as follows: the generating set $S$ consists of

- elements $x, y, s_{\dagger}, t$ and $u$;

- for each state $s_{i} \in S_{0}$ of type I or IX, an element $s_{i}$;

- for each state $s_{i} \in S_{0}$ of type VI, three elements $s_{i}, a_{i}, b_{i}$.

The alphabet $A$ consists of:

- four letters $0,1, \dagger_{1}$ and $\dagger_{2}$;

- for each state $s_{i} \in S_{0}$ of type I, a letter $\mathbf{i}_{1}$;

- for each state $s_{i} \in S_{0}$ of type IX, two letters $\mathbf{i}_{1}$ and $\mathbf{i}_{2}$;

- for each state $s_{i} \in S_{0}$ of type VI, five letters $\mathbf{i}_{1}, \mathbf{i}_{2}, \ldots, \mathbf{i}_{5}$.

The map $\Phi_{\mathrm{M}}: A \times S \rightarrow F_{S} \times A$ is given below, with $\epsilon$ denoting the empty word in $F_{S}$. Whenever a value of $\Phi_{\mathbf{M}}$ is unspecified, we take it to mean $\Phi_{\mathbf{M}}(a, s)=(s, a)$. 
- for the states $s_{\dagger}$ and $t, u$ we put

$$
\begin{aligned}
& \Phi_{\mathbf{M}}\left(0, s_{\dagger}\right)=\left(\epsilon, \dagger_{1}\right) ; \quad \Phi_{\mathbf{M}}\left(\dagger_{1}, x\right)=\left(\epsilon, \dagger_{1}\right) ; \quad \Phi_{\mathbf{M}}\left(\dagger_{1}, y\right)=\left(\epsilon, \dagger_{1}\right) ; \\
& \Phi_{\mathbf{M}}\left(\dagger_{1}, s_{\dagger}\right)=(\epsilon, 0) ; \quad \Phi_{\mathbf{M}}\left(\dagger_{2}, x\right)=\left(\epsilon, \dagger_{2}\right) ; \quad \Phi_{\mathbf{M}}\left(\dagger_{2}, y\right)=\left(\epsilon, \dagger_{2}\right) ; \\
& \Phi_{\mathbf{M}}\left(\dagger_{1}, t\right)=\left(\epsilon, \dagger_{2}\right) ; \quad \Phi_{\mathbf{M}}(0, u)=(u, 1) ; \quad \Phi_{\mathbf{M}}\left(\dagger_{2}, s_{\dagger}\right)=\left(\epsilon, \dagger_{2}\right) ; \\
& \Phi_{\mathbf{M}}\left(\dagger_{2}, t\right)=\left(\epsilon, \dagger_{1}\right) \quad \Phi_{\mathbf{M}}(1, u)=(u, 0) \text {. }
\end{aligned}
$$

- for all $g \in S \backslash\{u\}$ we put $\Phi_{\mathbf{M}}(1, g)=(\epsilon, 1)$, and for all $a \in A \backslash\{0,1\}$ we put $\Phi_{\mathbf{M}}(a, u)=(\epsilon, a)$.

- for each instruction $\left(s_{i}, m, n\right) \mapsto\left(s_{j}, m+1, n\right)$ of type I we put $\Phi_{\mathbf{M}}\left(0, s_{i}\right)=\left(s_{j}, \mathbf{i}_{1}\right) ; \quad \Phi_{\mathbf{M}}\left(\mathbf{i}_{1}, s_{i}\right)=(\epsilon, 0) ; \quad \Phi_{\mathbf{M}}\left(\mathbf{i}_{1}, x\right)=\left(x^{2}, \mathbf{i}_{1}\right) ; \quad \Phi_{\mathbf{M}}\left(\mathbf{i}_{1}, y\right)=\left(y, \mathbf{i}_{1}\right)$.

- for each instruction $\left(s_{i}, m, n\right) \mapsto\left(s_{j}, n, m\right)$ of type VI, $\Phi_{\mathbf{M}}(a, s)$ is written at position $(a, s)$ of the following table:

\begin{tabular}{cc|cccccc|} 
& & \multicolumn{5}{|c}{ input letter } \\
& 0 & $\mathbf{i}_{1}$ & $\mathbf{i}_{2}$ & $\mathbf{i}_{3}$ & $\mathbf{i}_{4}$ & $\mathbf{i}_{5}$ \\
\hline \multirow{2}{*}{} & $x$ & & $\left(x^{b_{i} x}, \mathbf{i}_{1}\right)$ & $\left(\epsilon, \mathbf{i}_{3}\right)$ & $\left(x, \mathbf{i}_{2}\right)$ & $\left(x^{2}, \mathbf{i}_{4}\right)$ & $\left(y, \mathbf{i}_{5}\right)$ \\
$\overrightarrow{0}$ & $y$ & & $\left(y^{x}, \mathbf{i}_{1}\right)$ & $\left(y, \mathbf{i}_{2}\right)$ & & $\left(y, \mathbf{i}_{4}\right)$ & $\left(x, \mathbf{i}_{5}\right)$ \\
$\vec{\rightleftarrows}$ & $s_{i}$ & $\left(a_{i} b_{i} x, \mathbf{i}_{1}\right)$ & $(\epsilon, 0)$ & & & \\
$\vec{\Xi}$ & $a_{i}$ & $\left(a_{i}, \mathbf{i}_{2}\right)$ & & $(\epsilon, 0)$ & & & \\
$\vec{d}$ & $b_{i}$ & & & $\left(b_{i}, \mathbf{i}_{4}\right)$ & $\left(a_{i}^{-1} s_{j}, \mathbf{i}_{5}\right)$ & $\left(\epsilon, \mathbf{i}_{2}\right)$ & $\left(\epsilon, \mathbf{i}_{3}\right)$ \\
\hline
\end{tabular}

- for each instruction $\left(s_{i}, m, n\right) \mapsto\left(m=0 ? s_{j}: s_{k}, \max (0, m-1), n\right)$ of type IX we put

$$
\begin{aligned}
& \Phi_{\mathbf{M}}\left(0, s_{i}\right)=\left(s_{k}, \mathbf{i}_{1}\right) ; \quad \Phi_{\mathbf{M}}\left(\mathbf{i}_{1}, x\right)=\left(s_{k}^{-1} s_{j} x, \mathbf{i}_{2}\right) ; \quad \Phi_{\mathbf{M}}\left(\mathbf{i}_{1}, y\right)=\left(y, \mathbf{i}_{1}\right) ; \\
& \Phi_{\mathbf{M}}\left(\mathbf{i}_{1}, s_{i}\right)=(\epsilon, 0) ; \quad \Phi_{\mathbf{M}}\left(\mathbf{i}_{2}, x\right)=\left(x^{-1} s_{j}^{-1} s_{k} x, \mathbf{i}_{1}\right) ; \quad \Phi_{\mathbf{M}}\left(\mathbf{i}_{2}, y\right)=\left(y, \mathbf{i}_{2}\right) .
\end{aligned}
$$

Theorem $\mathrm{A}^{\prime}$ follows from the undecidability of the halting problem for the Minsky machines (Proposition 2.2) and the following

Proposition 2.3. Consider the infinite sequence $W=0^{\infty}$. Then the Minsky machine $\mathbf{M}$ does not halt if and only if the action of $\left\langle\Phi_{\mathbf{M}}\right\rangle$ satisfies

$$
W^{\left(s_{*} x y\right) t\left(s_{*} x y\right)^{-1}}=W .
$$

Proof. We encode the states of $\mathbf{M}$ by elements of $F_{S}$. The word $\left(s_{i} x^{2^{m}} y^{2^{n}}\right) \alpha\left(s_{i} x^{2^{m}} y^{2^{n}}\right)^{-1}$ corresponds to the state $\left(s_{i}, m, n\right)$.

It is convenient to write $\Phi_{\mathbf{M}}(a, g)=\left(g^{\prime}, a^{\prime}\right)$ in the form $a \cdot g=g^{\prime} \cdot a^{\prime}$. In this manner, the computation of the functionally recursive action is given by a sequence of exchanges of letters with words in $F_{S}$. We check the following equalities:

If $\left(s_{i}, m, n\right) \rightarrow\left(s_{j}, m+1, n\right)$ is an instruction of type I, then

$$
0 \cdot\left(s_{i} x^{2^{m}} y^{2^{n}}\right) t\left(s_{i} x^{2^{m}} y^{2^{n}}\right)^{-1}=\left(s_{j} x^{2^{m+1}} y^{2^{n}}\right) t\left(s_{j} x^{2^{m+1}} y^{2^{n}}\right)^{-1} \cdot 0 .
$$

Indeed $0 \cdot s_{i} x^{2^{m}} y^{2^{n}}=s_{j} \cdot \mathbf{i}_{1} \cdot x^{2^{m}} y^{2^{n}}=s_{j} x^{2^{m+1}} y^{2^{n}} \cdot \mathbf{i}_{1}$; the claim follows from $\mathbf{i}_{1} \cdot t=t \cdot \mathbf{i}_{1}$ and the reverse $\mathbf{i}_{1} \cdot\left(s_{i} x^{2^{m}} y^{2^{n}}\right)^{-1}=\left(s_{j} x^{2^{m+1}} y^{2^{n}}\right)^{-1} \cdot 0$.

If $\left(s_{i}, m, n\right) \rightarrow\left(s_{j}, n, m\right)$ is an instruction of type VI, then

$$
0^{m+2} \cdot\left(s_{i} x^{2^{m}} y^{2^{n}}\right) t\left(s_{i} x^{2^{m}} y^{2^{n}}\right)^{-1}=\left(s_{j} x^{2^{n}} y^{2^{m}}\right) t\left(s_{j} x^{2^{n}} y^{2^{m}}\right)^{-1} \cdot 0^{m+2} .
$$

Indeed we first check $0 \cdot s_{i} x^{2^{m}} y^{2^{n}}=a_{i} b_{i} x\left(x^{b_{i} x}\right)^{2^{m}}\left(y^{x}\right)^{2^{n}} \cdot \mathbf{i}_{1}=a_{i} x^{2^{m}} b_{i} y^{2^{n}} x \cdot \mathbf{i}_{1}$. 
We obtained a word with two "blocks" of $x$ : the blocks $x^{2^{m}}$ and $x^{2^{0}}$. Each time a ' 0 ' letter is multiplied on the left of that word, the size of the first block will halve and the size of the second one will double: for $m, n, p \in \mathbb{N}$, we have

$$
\begin{gathered}
0 \cdot a_{i} x^{2 m} b_{i} y^{n} x^{p}=a_{i} x^{m} b_{i} y^{n} x^{2 p} \cdot \mathbf{i}_{4} \\
\text { so } 0^{m+1} \cdot s_{i} x^{2^{m}} y^{2^{n}}= \\
a_{i} x b_{i} y^{2^{n}} x^{2^{m}} \cdot\left(\mathbf{i}_{4}\right)^{m} \mathbf{i}_{1} \text {. Then } \\
0 \cdot a_{i} x b_{i} y^{2^{n}} x^{2^{m}}=a_{i}\left(a_{i}^{-1} s_{j}\right) x^{2^{n}} y^{2^{m}} \cdot \mathbf{i}_{5},
\end{gathered}
$$

so $0^{m+2} \cdot s_{i} x^{2^{m}} y^{2^{n}}=s_{j} x^{2^{n}} y^{2^{m}} \cdot \mathbf{i}_{5}\left(\mathbf{i}_{4}\right)^{m} \mathbf{i}_{1}$. Recalling that we have $a \cdot t=t \cdot a$ for all $a=\mathbf{i}_{1}, \ldots, \mathbf{i}_{5}$, the claim is proven.

If $(s, m, n) \rightarrow\left(m=0 ? s_{j}: s_{k}, \max (m-1,0), n\right)$ is an instruction of type IX, then if $m=0$ we have

$$
0 \cdot\left(s_{i} x^{2^{m}} y^{2^{n}}\right) t\left(s_{i} x^{2^{m}} y^{2^{n}}\right)^{-1}=\left(s_{j} x^{2^{m}} y^{2^{n}}\right) t\left(s_{j} x^{2^{m}} y^{2^{n}}\right)^{-1} \cdot 0
$$

while if $m>0$ we have

$$
0 \cdot\left(s_{i} x^{2^{m}} y^{2^{n}}\right) t\left(s_{i} x^{2^{m}} y^{2^{n}}\right)^{-1}=\left(s_{k} x^{2^{m-1}} y^{2^{n}}\right) t\left(s_{k} x^{2^{m-1}} y^{2^{n}}\right)^{-1} \cdot 0 .
$$

Indeed in the first case we have

$$
0 \cdot s_{i} x y^{2^{n}}=s_{k}\left(s_{k}^{-1} s_{j} x\right) y^{2^{n}} \cdot \mathbf{i}_{2},
$$

while in the second case we have

$$
0 \cdot s_{i} x^{2^{m}} y^{2^{n}}=s_{k}\left(s_{j}^{-1} s_{k} x \cdot x^{-1} s_{k}^{-1} s_{j} x\right)^{2^{m-1}} y^{2^{n}} \cdot \mathbf{i}_{1}=s_{k} x^{2^{m-1}} y^{2^{n}} \cdot \mathbf{i}_{1} .
$$

Recalling that we have $a \cdot t=t \cdot a$ for all $a=\mathbf{i}_{1}, \mathbf{i}_{2}$, the claim is proven.

From (2.1)-(2.4) it follows that if $\mathbf{M}$ does not halt then $W^{\left(s_{*} x y\right) t\left(s_{*} x y\right)^{-1}}=W$. Conversely, if $\mathbf{M}$ halts then there exist $k, m, n \in \mathbb{N}$ such that

$$
0^{k} \cdot\left(s_{*} x y\right) t\left(s_{*} x y\right)^{-1}=\left(s_{\dagger} x^{2^{m}} y^{2^{n}}\right) t\left(s_{\dagger} x^{2^{m}} y^{2^{n}}\right)^{-1} \cdot 0^{k} .
$$

Then

$$
0 \cdot s_{\dagger} x^{2^{m}} y^{2^{n}} t\left(s_{\dagger} x^{2^{m}} y^{2^{n}}\right)^{-1}=\dagger_{1} \cdot t\left(s_{\dagger} x^{2^{m}} y^{2^{n}}\right)^{-1}=\dagger_{2} \cdot\left(s_{\dagger} x^{2^{m}} y^{2^{n}}\right)^{-1}=\dagger_{2} \cdot
$$

In that case, we have $W^{\left(s_{*} x y\right) t\left(s_{*} x y\right)^{-1}}=0^{k} \dagger_{2} 0^{\infty} \neq W$.

The computations are best carried on $\Phi_{\mathbf{M}}$ 's dual Moore diagram $\Delta$, see Figure 2 : this is the directed labeled graph with vertex set $A$ and with for all $a \in A, s \in S$ an edge from $a$ to $b$ labeled $(s, t)$ whenever $\Phi_{\mathbf{M}}(a, s)=(t, b)$. One checks an equality ' $\Phi_{\mathbf{M}}(a, s)=(t, b)$ ' by finding in $\Delta$ a path starting at $a$ with input label $s$; the endpoint of the path is $b$, and the output label is $t$.

2.2. Proof of Theorem $\mathbf{A}$. We have not yet used the letter 1 and the state $u$ of $\Phi_{\mathbf{M}}$. Theorem $\AA$ follows now from the following

Proposition 2.4. The Minsky machine $\mathbf{M}$ halts if and only if

$$
\left[\left(s_{*} x y\right) t\left(s_{*} x y\right)^{-1}, u\right] \neq 1 \text { in }\left\langle\Phi_{\mathbf{M}}\right\rangle .
$$

Proof. The element $u$ acts on $A^{\omega}$ as follows: it scans $X \in A^{\omega}$ for its longest prefix in $\{0,1\}^{*}$, and exchanges all 0 and 1 in that prefix. Write $g=\left(s_{*} x y\right) t\left(s_{*} x y\right)^{-1}$; from Proposition 2.3 we know that $g$ fixes $0^{\infty}$ if and only if $\mathbf{M}$ does not halt.

Assume first that $\mathbf{M}$ does not halt; then $g$ in fact also fixes $\{0,1\}^{\infty}$, so the supports of $g$ and $u$ are disjoint and $[g, u]=1$ in $\left\langle\Phi_{\mathbf{M}}\right\rangle$. 


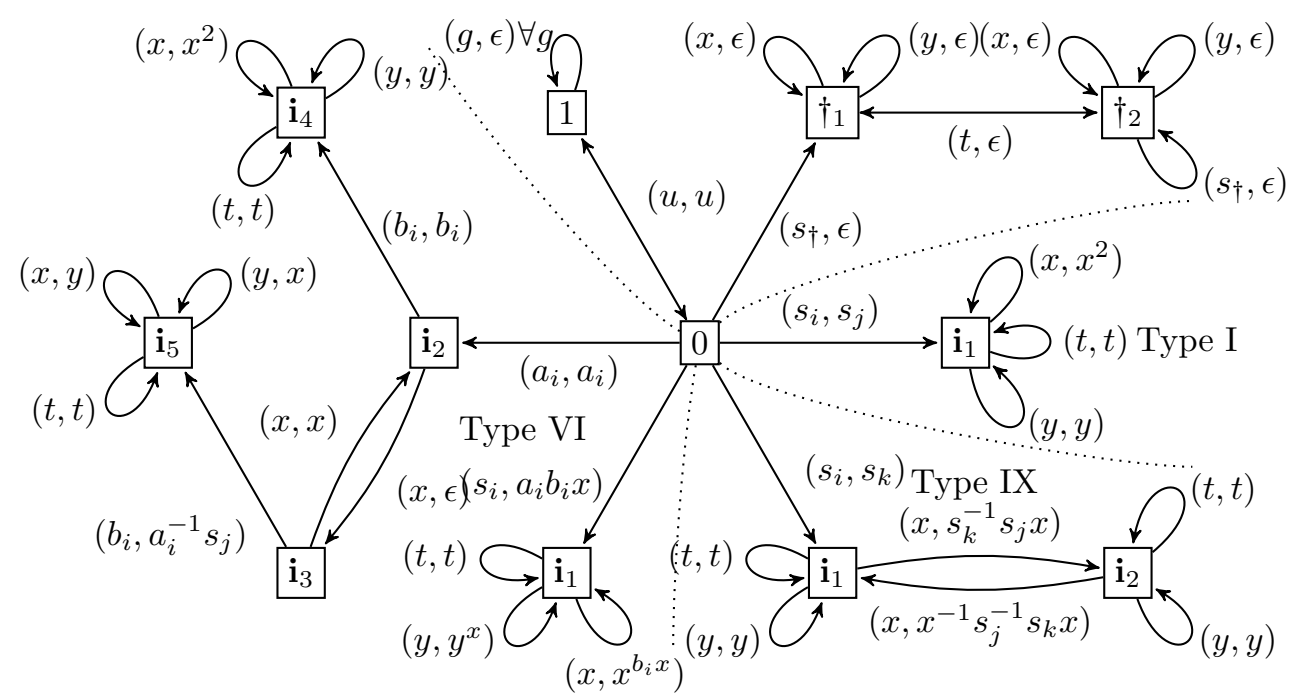

Figure 2. The dual Moore diagram of $\Phi_{\mathbf{M}}$, used in the proof of Theorem $\mathrm{A}$

Assume next that $\mathbf{M}$ does halt; without loss of generality, we may assume $\mathbf{M}$ does not stop immediately, so there is $k \geqslant 1$ such that $\left(0^{k+1}\right)^{g}=0^{k} \dagger_{2}$. Since $\left(0^{k+1}\right)^{u}=1^{k+1}$ and $\left(0^{k} \dagger_{2}\right)^{u}=1^{k} \dagger_{2}$ and $\left(1^{k+1}\right)^{g}=1^{k+1}$ and $\left(1^{k} \dagger_{2}\right)^{g}=1^{k} \dagger_{2}$, the commutator $[g, u]$ acts as a 2 -2-cycle $\left(0^{k+1}, 0^{k} \dagger_{2}\right)\left(1^{k+1}, 1^{k} \dagger_{2}\right)$ and in particular $[g, u] \neq 1$ in $\left\langle\Phi_{\mathbf{M}}\right\rangle:$

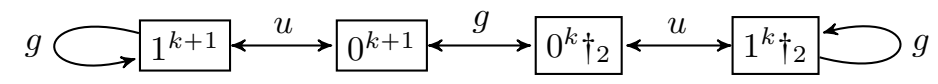

2.3. Proof of Theorem $\mathbf{A}^{\prime \prime}$. Consider a universal Minsky machine $\mathbf{M}_{u}$, namely one that emulates arbitrary Turing machines encoded in an integer $n$, when started in state $\left(s_{*}, 0, n\right)$. The set of $2^{n}$ such that $\mathbf{M}_{u}$ halts when started in state $\left(s_{*}, 0, n\right)$ is not recursive, see Proposition 2.2. Therefore, Theorem $\mathrm{A}^{\prime \prime}$ follows by considering in the group $\left\langle\Phi_{\mathbf{M}_{u}}\right\rangle$ the elements $\left[\left(s_{*} x y^{2^{n}}\right) t\left(s_{*} x y^{2^{n}}\right)^{-1}, u\right]$; this set of words is recursive, but the subset of those that equal $1 \mathrm{in}\left\langle\Phi_{\mathbf{M}_{u}}\right\rangle$ is not recursive.

\section{Automata groups and Minsky machines}

3.1. Proof of Theorem B, Let $\mathbf{M}$ by a Minsky machine with stateset $S_{0}$. Without loss of generality, we assume that all instructions of $\mathbf{M}$ are of type III,IV,V,VII,VIII, as defined in the beginning of Section 2 .

$$
S_{0}=S_{\mathrm{III}} \sqcup S_{\mathrm{IV}} \sqcup S_{\mathrm{V}} \sqcup S_{\mathrm{VII}} \sqcup S_{\mathrm{VIII}} \sqcup\left\{s_{\dagger}\right\}
$$

We consider the transducer with stateset $S:=S_{0}^{ \pm 1} \sqcup\left\{\epsilon, x, x^{-1}, y, y^{-1}\right\}$ and alphabet

$$
A=\left\{\mathrm{III}_{i}, \mathrm{IV}_{i}, \mathrm{~V}_{i}, \mathrm{VII}_{j}, \mathrm{VIII}_{j} \mid i=1,2, \overline{1}, \overline{2} ; j=1, \ldots, 4, \overline{1}, \ldots, \overline{4}\right\} .
$$


The structure of the transducer is given by its map $\Phi_{\mathrm{M}}: A \times S \rightarrow S \times A$, first described as a table, with $\Phi_{\mathbf{M}}(a, s)$ at position $(a, s)$. The state $\epsilon$ is the identity, and $\Phi_{\mathbf{M}}(a, \epsilon)=(\epsilon, a)$ for all $a \in A$.

For all instructions $(s, m, n) \mapsto\left(s^{\prime}, m+1, n+1\right)$ of type III and for all $t \in S_{0} \backslash S_{\text {III }}$ we have

\begin{tabular}{|c|c|c|c|c|c|}
\hline & \multicolumn{4}{|c|}{ input letter } \\
\hline & & $\mathrm{III}_{1}$ & $\mathrm{III}_{2}$ & $\mathrm{III}_{\overline{1}}$ & $\mathrm{III}_{\overline{2}}$ \\
\hline & & $\left(x, \mathrm{III}_{1}\right)$ & $\left(x, \mathrm{III}_{2}\right)$ & $\left(x^{-1}, \mathrm{III}_{\overline{1}}\right)$ & $\left(x^{-1}, \mathrm{III}_{2}\right)$ \\
\hline 需 & $y$ & $\left(y, \mathrm{III}_{1}\right)$ & $\left(y, \mathrm{III}_{2}\right)$ & $\left(y^{-1}, \mathrm{III}_{\overline{1}}\right)$ & $\left(y^{-1}, \mathrm{III}_{\overline{1}}\right)$ \\
\hline & $s$ & $\left(s^{\prime}, \mathrm{III}_{2}\right)$ & $\left(\epsilon, \mathrm{III}_{1}\right)$ & $\left(\epsilon, \mathrm{III}_{\overline{2}}\right)$ & $\left(\left(s^{\prime}\right)^{-1}, \mathrm{III}_{\overline{1}}\right)$ \\
\hline & $t$ & $\left(\epsilon, \mathrm{III}_{\overline{1}}\right)$ & $\left(\epsilon, \mathrm{III}_{\overline{2}}\right)$ & $\left(\epsilon, \mathrm{III}_{1}\right)$ & $\left(\epsilon, \mathrm{III}_{2}\right)$ \\
\hline
\end{tabular}

For all instructions $(s, m, n) \mapsto\left(s^{\prime}, m-1, n\right)$ of type IV and for all $t \in S_{0} \backslash S_{\mathrm{IV}}$, we have

\begin{tabular}{|c|c|c|c|c|c|}
\hline & \multicolumn{4}{|c|}{ input letter } \\
\hline & & $\mathrm{IV}_{1}$ & $\mathrm{IV}_{2}$ & $\mathrm{IV}_{\overline{1}}$ & $\mathrm{IV}_{\overline{2}}$ \\
\hline & & $\left(x, \mathrm{IV}_{2}\right)$ & $\left(\epsilon, \mathrm{IV}_{1}\right)$ & $\left(\epsilon, \mathrm{IV}_{\overline{2}}\right)$ & $\left(x^{-1}, I_{\overline{1}}\right)$ \\
\hline & $y$ & $\left(y, \mathrm{IV}_{1}\right)$ & $\left(y, \mathrm{IV}_{2}\right)$ & $\left(y^{-1}, \mathrm{IV}_{\overline{1}}\right)$ & $\left(y^{-1}, I_{\overline{2}}\right)$ \\
\hline & $s$ & $\left(s^{\prime}, \mathrm{IV}_{1}\right)$ & $\left(s^{\prime}, \mathrm{IV}_{2}\right)$ & $\left(\left(s^{\prime}\right)^{-1}, \mathrm{IV}_{\overline{1}}\right)$ & $\left(\left(s^{\prime}\right)^{-1}, \mathrm{IV}_{\overline{2}}\right)$ \\
\hline & $t$ & $\left(\epsilon, \mathrm{IV}_{\overline{\mathrm{I}}}\right)$ & $\left(\epsilon, \mathrm{IV}_{\overline{2}}\right)$ & $\left(\epsilon, \mathrm{IV}_{1}\right)$ & $\left(\epsilon, \mathrm{IV}_{2}\right)$ \\
\hline
\end{tabular}

The same applies for an instruction of type $\mathrm{V}$, with the roles of $x, y$ switched.

For an instruction $(s, m, n) \mapsto\left(m=0 ? s^{\prime}: s^{\prime \prime}, m, n\right)$ of type VII and for all $t \in S_{0} \backslash S_{\mathrm{VII}}$, we have

\begin{tabular}{|c|c|c|c|c|c|c|c|c|c|}
\hline & \multicolumn{8}{|c|}{ at letter } \\
\hline & & $\mathrm{VII}_{1}$ & $\mathrm{VII}_{2}$ & $\mathrm{VII}_{3}$ & $\mathrm{VII}_{4}$ & $\mathrm{VII}_{\overline{1}}$ & $\mathrm{VII}_{\overline{2}}$ & $\mathrm{VII}_{\overline{3}}$ & $\mathrm{VII}_{\overline{4}}$ \\
\hline & & $\left(x, \mathrm{VII}_{4}\right)$ & $\left(\epsilon, \mathrm{VII}_{3}\right)$ & $\left(\epsilon, \mathrm{VII}_{2}\right)$ & $\left(x, \mathrm{VII}_{1}\right)$ & $\left(x^{-1}, \mathrm{VII}_{\overline{4}}\right)$ & $\left(\epsilon, \mathrm{VII}_{\overline{3}}\right)$ & $\left(\epsilon, \mathrm{VII}_{\overline{2}}\right)$ & $\left(x^{-1}, \mathrm{VII}_{\overline{1}}\right)$ \\
\hline & $y$ & $\left(y, \mathrm{VII}_{1}\right)$ & $\left(\epsilon, \mathrm{VII}_{2}\right)$ & $\left(\epsilon, \mathrm{VII}_{3}\right)$ & $\left(y, \mathrm{VII}_{4}\right)$ & $\left(y^{-1}, \mathrm{VII}_{\overline{1}}\right)$ & $\left(\epsilon, \mathrm{VII}_{2}\right)$ & $\left(\epsilon, \mathrm{VII}_{3}\right)$ & $\left(y^{-1}, \mathrm{VII}_{\overline{4}}\right)$ \\
\hline & $s$ & $\left(\epsilon, \mathrm{VII}_{2}\right)$ & $\left(s^{\prime \prime}, \mathrm{VII}_{1}\right)$ & $\left(s^{\prime}, \mathrm{VII}_{4}\right)$ & $\left(\epsilon, \mathrm{VII}_{\overline{4}}\right)$ & $\left(\left(s^{\prime \prime}\right)^{-1}, \mathrm{VII}_{\overline{2}}\right)$ & $\left(\epsilon, \mathrm{VII}_{\overline{1}}\right)$ & $\left(\epsilon, \mathrm{VII}_{3}\right)$ & $\left(\left(s^{\prime}\right)^{-1}, \mathrm{VII}_{\overline{3}}\right)$ \\
\hline & $t$ & $\left(\epsilon, \mathrm{VII}_{\overline{\mathrm{T}}}\right)$ & $\left(\epsilon, \mathrm{VII}_{\overline{2}}\right)$ & $\left(\epsilon, \mathrm{VII}_{\overline{3}}\right)$ & $\left(\epsilon, \mathrm{VII}_{\overline{4}}\right)$ & $\left(\epsilon, \mathrm{VII}_{1}\right)$ & $\left(\epsilon, \mathrm{VII}_{2}\right)$ & $\left(\epsilon, \mathrm{VII}_{3}\right)$ & $\left(\epsilon, \mathrm{VII}_{4}\right)$ \\
\hline
\end{tabular}

The same applies for an instruction of type VIII, with the roles of $x, y$ switched.

Note that $s+$ is treated as a state $t$ in all tables above.

Theorem $\mathrm{B}$ follows from the undecidability of the halting problem for Minsky machines, and the following

Proposition 3.1. The Minsky machine $\mathbf{M}$ constructed above halts if and only if the element $s_{*} x y$ has finite order in $\left\langle\Phi_{\mathbf{M}}\right\rangle$.

Proof. Set $G=\left\langle\Phi_{\mathbf{M}}\right\rangle$. For $g \in G$, denote by $C(g)$ its symmetric conjugacy class:

$$
C(g):=\left\{g^{ \pm x} \mid x \in G\right\} \text {. }
$$

Given a symmetric conjugacy class $C$, choose a representative $g$ in it, let $A=$ $A_{1} \sqcup \cdots \sqcup A_{\ell}$ be the decomposition of $A$ into cycles for the action of $g$, and choose representatives $a_{i} \in A_{i}$. We have $\bar{\Phi}_{\mathbf{M}}\left(a_{i}, g^{\# A_{i}}\right)=\left(h_{i}, a_{i}\right)$ for some $h_{i} \in G$, and it is easy to see that the collection of symmetric conjugacy class $\left\{C\left(h_{i}\right) \mid i=1, \ldots, \ell\right\}$ is independent of the choice of $g$ and the $a_{i}$.

We construct an integer-labeled, directed graph 1 whose vertices are symmetric conjugacy classes in $G$; for a conjugacy class $C$ as above, there are $\ell$ edges starting at $C$, ending respectively at $C\left(h_{1}\right), \ldots, C\left(h_{\ell}\right)$ with labels $\# A_{1}, \ldots, \# A_{\ell}$.

\footnotetext{
${ }^{1}$ This graph essentially appears in the solution of $[3$ to the order problem in bounded automata.
} 
Lemma 3.2. For $g \in G$, its order (in $\mathbb{N} \cup\{\infty\}$ ) is the least common multiple, along all paths starting at $C(g)$, of the product of the labels along the path.

Proof. Consider a path starting at $C(g)$, with labels $n_{1}, \ldots, n_{s}$, and going through vertices $C\left(g_{1}\right), \ldots, C\left(g_{s}\right)$. Then $g$ has an orbit of length $n_{1}$ on $A$, so the order of $g$ is a multiple of $n_{1}$. Furthermore, $g^{n_{1}}$ fixes that orbit, and acts as $g_{1}$ on sequences that start by that orbit. Recursively, the order of $g_{1}$ is a multiple of $n_{2} \cdots n_{s}$, so the order of $g$ is a multiple of $n_{1} \cdots n_{s}$. In particular, if there are paths with arbitrarily large product of labels then $g$ has infinite order.

Conversely, if $g$ has infinite order then there are arbitrarily long orbits of $g$ on $A^{*}$, so there are paths with arbitrarily large product of labels; and if $m$ be the least common multiple of all path labels then all edges on paths starting at $C\left(g^{m}\right)$ are labeled 1 so $g^{m}$ fixes every sequence and therefore $g^{m}=1$.

Let us compute the subgraph spanned by $C\left(s_{*} x y\right)$. For the computations, it is helpful to picture the operation of the transducer $\Phi_{\mathbf{M}}$ by means of its dual Moore diagram $\Delta$, see Figure 3. Given $g \in G$, we compute all primitive cycles in $\Delta$ whose input label is a power of $g$, and read the corresponding output label; these are the $h_{i}$ in the map on symmetric conjugacy classes $C(g) \rightsquigarrow\left\{C\left(h_{i}\right)\right\}$.

We first note, by direct inspection, that $x$ and $y$ commute in $G$. This follows by tracing the path $x^{-1} y^{-1} x y$ in the graphs above, and noting that they always induce the trivial permutation of $A$ with output either trivial or conjugate to $\left(x^{-1} y^{-1} x y\right)^{ \pm 1}$.

We now claim that, if $(s, m, n) \rightarrow\left(s^{\prime}, m^{\prime}, n^{\prime}\right)$ is a transition of the machine $\mathbf{M}$, then the conjugacy class $C\left(s x^{2^{m}} y^{2^{n}}\right)$ has at least one arrow to $C\left(s^{\prime} x^{2^{m^{\prime}}} y^{2^{n^{\prime}}}\right)$, and possibly other arrows, all of them to $C(1)$. We also claim that if $s$ is not of type IV or $\mathrm{V}$, then arrows to $C\left(s^{\prime} x^{2^{m^{\prime}}} y^{2^{n^{\prime}}}\right)$ are with labels $>1$; and all arrows from $C\left(s_{\dagger} x^{2^{m}} y^{2^{n}}\right)$ are arrows to $C(1)$. We see that if the machine halts then every path starting at $C\left(s_{*} x y\right)$ has only a finite number of labels $>1$, and this shows that the order of $s_{*} x y$ is finite.

On the other hand, if the machine does not halt then there is an path with infinitely many labels $>1$ (because no Minsky machine can decrease its counters infinitely many times in a row) so $s_{*} x y$ has infinite order.

Note that our transducer has the property $\Phi_{\mathbf{M}}\left(L_{\bar{i}}, g\right)=\Phi_{\mathbf{M}}\left(L_{i}, g^{-1}\right)$, for all $g \in S$ and all $L \in\{$ III, IV, V, VII, VIII $\}$. Also note that $\Phi_{\mathbf{M}}\left(L_{i}, t\right)=\Phi_{\mathbf{M}}\left(L_{i}, t^{-1}\right)=\left(\epsilon, L_{\bar{i}}\right)$ whenever $t$ is any instruction not of type $L$.

Using this, we can prove that if $t$ is not of type $L$, then $t g_{n} g_{n-1} \cdots g_{1} t g_{1} g_{2} \cdots g_{n}$ fixes the orbit $\left\{L_{i}\right\}$ with output $\epsilon$.

Indeed, $t g_{n} g_{n-1} \cdots g_{1} t g_{1} g_{2} \cdots g_{n}=\left(g_{n} t g_{n} t^{-1} \cdot t g_{n-1} g_{n-2} \cdots g_{1} t g_{1} g_{2} \cdots g_{n-1}\right)^{g_{n}}$, and we use induction on $n$. It follows that $\left(t x^{m} y^{n}\right)^{2}$ fixes $L_{i}$ with outputs $\epsilon$, i.e., there is an arrow from $C\left(t x^{m} y^{n}\right)$ to $C(1)$ with label 2 .

Let us first restrict to the orbit $\left\{\mathrm{III}_{i}\right\}$ of $G$ on $A$. We consider $g:=s x^{m} y^{n}$ with $s$ an instruction of type III. It acts as a product of two cycles $\left(\mathrm{III}_{1}, \mathrm{III}_{2}\right)\left(\mathrm{III}_{\overline{1}}, \mathrm{III}_{\overline{2}}\right)$; the output label of $g^{2}$ on the first cycle, starting at $\mathrm{III}_{1}$, is $s^{\prime} x^{m} y^{n} \epsilon x^{m} y^{n}=s^{\prime} x^{2 m} y^{2 n}$, and the output of $g^{2}$ starting on the second cycle at $\mathrm{III}_{\overline{1}}$ is $\epsilon x^{-m} y^{-n}\left(s^{\prime}\right)^{-1} x^{-m} y^{-n} \in$ $C\left(s^{\prime} x^{2 m} y^{2 n}\right)$. There are therefore two arrows from $C\left(s x^{2^{m}} y^{2^{n}}\right)$ to $C\left(s^{\prime} x^{2^{m+1}} y^{2^{n+1}}\right)$, as required. We do not consider $g:=t x^{m} y^{n}$ with $t$ an instruction of different type or $s_{\dagger}$, because it was considered above (there are some arrows to $C(1)$ with labels 2). 
THE WORD AND ORDER PROBLEMS FOR SELF-SIMILAR AND AUTOMATA GROUPS 11
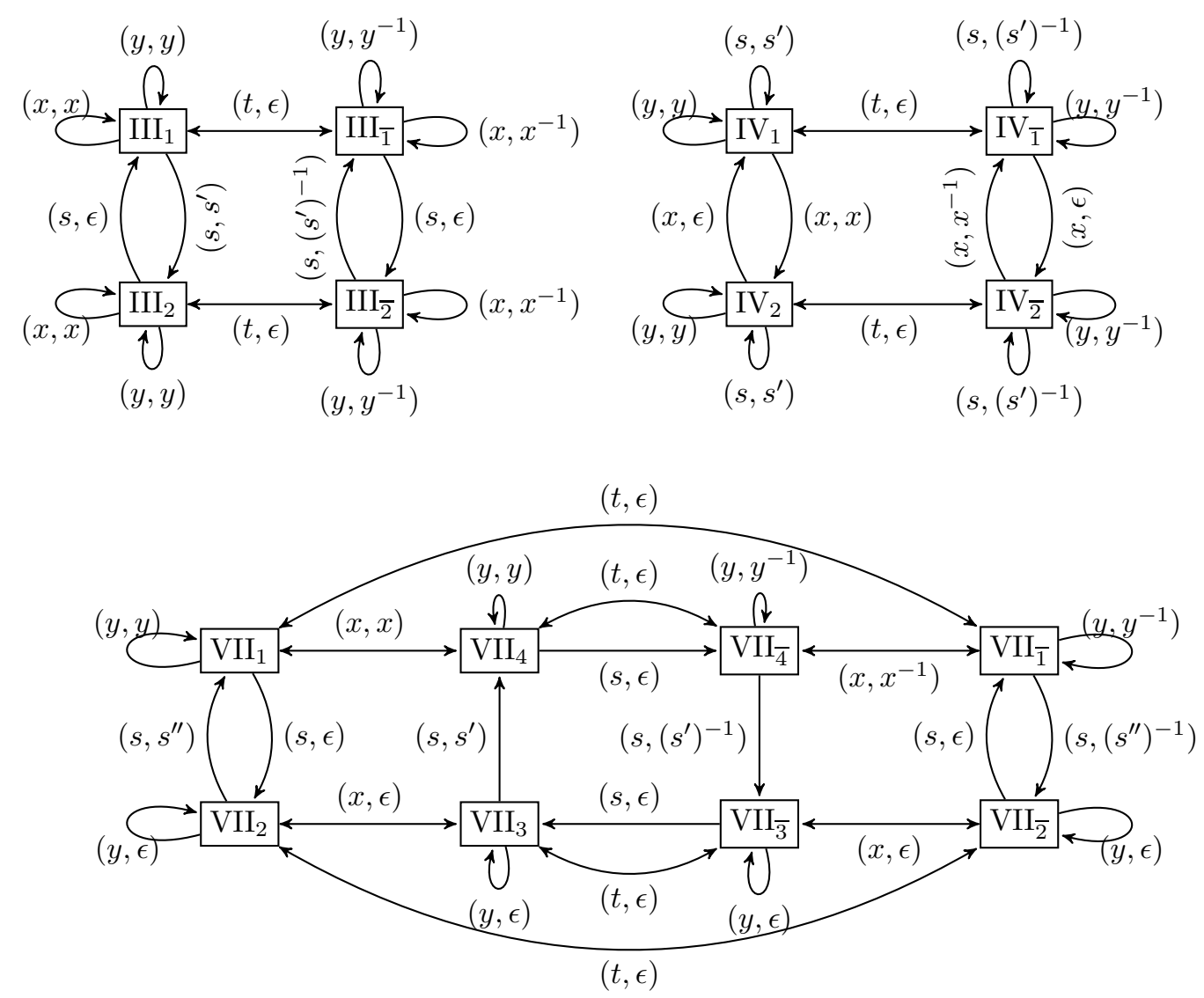

Figure 3 . The dual Moore diagram of $\Phi_{\mathbf{M}}$, used in the proof of Theorem B

We restrict next to the orbit $\left\{\mathrm{IV}_{i}\right\}$ of $G$ and consider the case $g=s x^{2 m} y^{n}$. (We do not need to consider cases $g=s x^{2 m+1} y^{n}$ or $g=t x^{m} y^{n}$, for the first because we suppose that if $m=0$ then $\mathbf{M}$ does not perform an instruction of type IV, and for the second because it was already considered above.)

An element $g=s x^{2 m} y^{n}$ fixes $\mathrm{IV}_{1}, \mathrm{IV}_{2}, \mathrm{IV}_{\overline{1}}$ and $\mathrm{IV}_{\overline{2}}$, its outputs are respectively $s^{\prime}(x \epsilon)^{m} y^{n}=s^{\prime} x^{m} y^{n}, s^{\prime} x^{m} y^{n},\left(s^{\prime}\right)^{-1} x^{-m} y^{-n}$ and $\left(s^{\prime}\right)^{-1} x^{-m} y^{-n}$. Hence there are 4 arrows from $C\left(s x^{2^{m}} y^{2^{n}}\right)$ to $C\left(s^{\prime} x^{2^{m-1}} y^{2^{n}}\right)$, all with labels 1 .

We restrict next to the orbit $\left\{\mathrm{VII}_{i}\right\}$ of $G$ and perform the same computations, the result is in the following table:

\begin{tabular}{c|c|l|}
$g \in G$ & cycles of $g$ & output, starting at first element of the cycle \\
\hline \multirow{3}{*}{$s x^{2 m} y^{n}$} & $\left(\mathrm{VII}_{1}, \mathrm{VII}_{2}\right)$ & $\epsilon s^{\prime \prime} x^{2 m} y^{n}$ \\
& $\left(\mathrm{VII}_{3}, \mathrm{VII}_{4}, \mathrm{VII}_{\overline{4}}, \mathrm{VII}_{\overline{3}}\right)$ & $s^{\prime} x^{2 m} y^{n} \epsilon x^{-2 m} y^{-n}\left(s^{\prime}\right)^{-1} \epsilon=1$ \\
& $\left(\mathrm{VII}_{\overline{1}}, \mathrm{VII}_{\overline{2}}\right)$ & $\left(s^{\prime \prime}\right)^{-1} \epsilon x^{-2 m} y^{-n}$ \\
\hline \multirow{3}{*}{$s x^{2 m+1} y^{n}$} & $\left(\mathrm{VII}_{1}, \mathrm{VII}_{3}\right)$ & $\epsilon^{\prime} x^{2 m+1} y^{n}$ \\
& $\left(\mathrm{VII}_{2}, \mathrm{VII}_{4}, \mathrm{VII}_{\overline{1}}, \mathrm{VII}_{\overline{3}}\right)$ & $s^{\prime \prime} x^{2 m+1} y^{n} \epsilon x^{-2 m-1} y^{-n}\left(s^{\prime \prime}\right)^{-1} \epsilon=1$ \\
& $\left(\mathrm{VII}_{\overline{2}}, \mathrm{VII}_{\overline{4}}\right)$ & $\epsilon x^{-2 m-1} y^{-n}\left(s^{\prime}\right)^{-1} \epsilon$ \\
\hline
\end{tabular}


If $m>0$ then there are therefore two arrows from $C\left(s x^{2^{m}} y^{2^{n}}\right)$ to $C\left(s^{\prime \prime} x^{2^{m}} y^{2^{n}}\right)$ with labels 2 and an arrow to $C(1)$ with label 4 ; if $m=0$ then there are two arrows from $C\left(s x^{2^{m}} y^{2^{n}}\right)$ to $C\left(s^{\prime \prime} x^{2^{m}} y^{2^{n}}\right)$ with label 4 and an arrow to $C(1)$ with label 4 .

The orbits $\left\{\mathrm{V}_{i}\right\}$ and $\left\{\mathrm{VIII}_{i}\right\}$ are investigated in the same way as $\left\{\mathrm{IV}_{i}\right\}$ and $\left\{\mathrm{VII}_{i}\right\}$ respectively.

3.2. Proof of Theorem $\mathbf{B}^{\prime \prime}$. Consider a universal Minsky machine $\mathbf{M}_{u}$, namely one that emulates arbitrary Turing machines encoded in an integer $n$, when started in state $\left(s_{*}, 0, n\right)$. The set of $2^{n}$ such that $\mathbf{M}_{u}$ halts when started in state $\left(s_{*}, 0, n\right)$ is not recursive, see Proposition 2.2 . Therefore, Theorem $\mathrm{B}^{\prime \prime}$ follows by considering in the group $\left\langle\Phi_{\mathbf{M}_{u}}\right\rangle$ the elements $s=s_{*} x$ and $t=y$.

3.3. Proof of Theorem $\mathbf{B}^{\prime}$. Let $\mathbf{M}$ be a Minsky machine with stateset $S_{0}$. Without loss of generality, we assume that all instructions are of type III,IX,X.

We associate to it the transducer with stateset $Q:=S_{0} \sqcup\{\epsilon, x, y\}$ and alphabet

$$
A=\left\{0, \mathrm{III}_{i}, \mathrm{IX}_{j}, \mathrm{X}_{j} \mid i=1,2 ; j=1, \ldots, 4\right\} .
$$

The structure of the transducer is given by its map $\Phi_{\mathbf{M}}: A \times Q \rightarrow Q \times A$.

The state $\epsilon$ is the identity, and $\Phi_{\mathbf{M}}(a, \epsilon)=(\epsilon, a)$ for all $a \in A$.

- for all instructions $\left(s_{i}, m, n\right) \mapsto\left(s_{i}^{\prime}, m+1, n+1\right)$ of type III we have

\begin{tabular}{|c|c|c|c|c|}
\hline & \multicolumn{3}{|c|}{ input letter } \\
\hline & & 0 & $\mathrm{III}_{1}$ & $\mathrm{III}_{2}$ \\
\hline 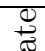 & $x$ & $(\epsilon, 0)$ & $\left(x, \mathrm{III}_{1}\right)$ & $\left(x, \mathrm{III}_{2}\right)$ \\
\hline$\frac{\pi}{0}$ & $y$ & $(\epsilon, 0)$ & $\left(y, \mathrm{III}_{1}\right)$ & $\left(y, \mathrm{III}_{2}\right)$ \\
\hline.$\Xi$ & $s_{i}$ & $\left(s_{i}^{\prime}, \mathrm{III}_{1}\right)$ & $\left(\epsilon, \mathrm{III}_{2}\right)$ & $(\epsilon, 0)$ \\
\hline
\end{tabular}

and every instruction $t$ of another type acts as $\Phi_{\mathbf{M}}\left(t, \mathrm{III}_{\ell}\right)=\left(\mathrm{III}_{\ell}, t\right)$.

- for all instructions $\left(s_{j}, m, n\right) \mapsto m=0$ ? $\left(s_{j}^{\prime}, m, n\right):\left(s_{j}^{\prime \prime}, m-1, n\right)$ of type IX we have

\begin{tabular}{|c|c|c|c|c|c|c|}
\hline & \multicolumn{5}{|c|}{ input letter } \\
\hline$\stackrel{ \pm}{ \pm}$ & $x$ & $(\epsilon, 0)$ & $\left(\epsilon, \mathrm{IX}_{2}\right)$ & $\left(\epsilon, \mathrm{IX}_{1}\right)$ & $\left(x, \mathrm{IX}_{4}\right)$ & $\overline{\left(\epsilon, \mathrm{IX}_{3}\right)}$ \\
\hline$\frac{i}{0}$ & $y$ & $(\epsilon, 0)$ & $\left(\epsilon, \mathrm{IX}_{1}\right)$ & $\left(\epsilon, \mathrm{IX}_{2}\right)$ & $\left(y, \mathrm{IX}_{3}\right)$ & $\left(y, \mathrm{IX}_{4}\right)$ \\
\hline.$\nexists$ & $s_{j}$ & $\left(\epsilon, \mathrm{IX}_{1}\right)$ & $\left(s_{j}^{\prime \prime}, \mathrm{IX}_{4}\right)$ & $\left(s_{j}^{\prime}, \mathrm{IX}_{3}\right)$ & $\left(s_{j}^{\prime}, \mathrm{IX}_{2}\right)$ & $(\epsilon, 0)$ \\
\hline
\end{tabular}

and every instruction $t$ of another type acts as $\Phi_{\mathbf{M}}\left(\operatorname{IX}_{\ell}, t\right)=\left(t, \operatorname{IX}_{\ell}\right)$.

- The same applies for every instruction $\left(s_{k}, m, n\right) \mapsto n=0$ ? $\left(s_{k}^{\prime}, m, n\right)$ : $\left(s_{k}^{\prime \prime}, m, n-1\right)$ of type $\mathrm{X}$, with the roles of $x$ and $y$ switched.

- for all $a \in A$ we have $\Phi_{\mathbf{M}}\left(a, s_{\dagger}\right)=(\epsilon, a)$.

We claim that the orbit of $0^{\infty}$ under $s_{*} x y$ is finite if and only if the machine $\mathbf{M}$ stops.

Set $G=\left\langle\Phi_{\mathbf{M}}\right\rangle$. We construct an integer-labeled, directed graph whose vertices are elements of $G$. For $g \in G$ consider its action on $A$ and the minimal $p_{g}$ such that $g^{p_{g}}$ fixes 0 , i.e., $0 \cdot g^{p_{g}}=g^{\prime} \cdot 0$. In our graph we put an edge $g \rightarrow g^{\prime}$ with label $p_{g}$ on it.

The size of the the orbit of $0^{\infty}$ under $s_{*} x y$ is a finite number or $\infty$ and it is equal to product of the labels along the path starting at $s_{*} x y$.

We claim that for any instruction $(s, m, n) \rightarrow\left(s^{\prime}, m^{\prime}, n^{\prime}\right)$ there is an edge from $s x^{2^{m}} y^{2^{n}}$ to $s^{\prime} x^{2^{m^{\prime}}} y^{2^{n^{\prime}}}$ with label 3 , and an edge from $s_{\dagger} x^{2^{m}} y^{2^{n}}$ to 1 . This is checked on the dual Moore diagram of $\Phi_{\mathbf{M}}$, see Figure 4 . 


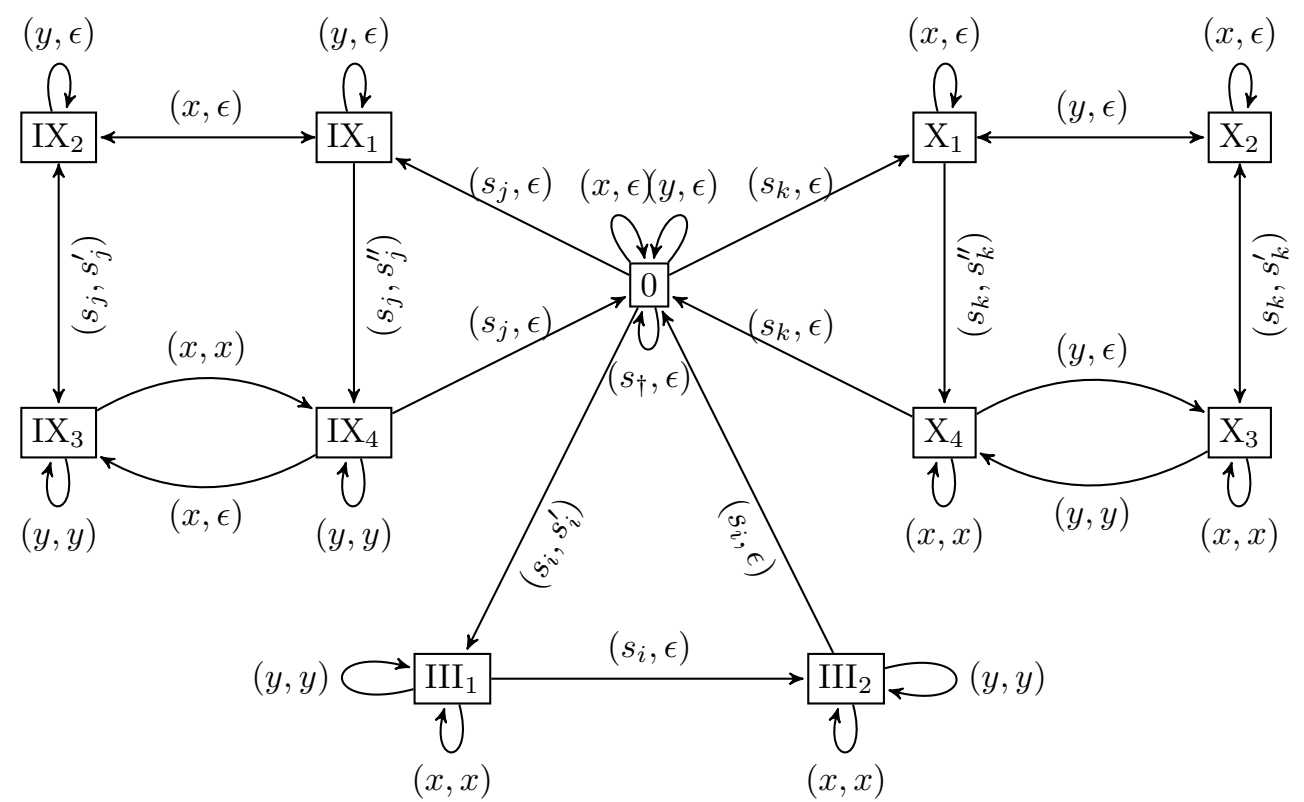

Figure 4 . The dual Moore diagram of $\Phi_{\mathbf{M}}$, used in the proof of Theorem $B^{\prime}$

We first note that $x$ and $y$ commute in $G$. If $g=s_{i} x^{m} y^{n}$ and $s_{i}$ is an instruction of type III, then the orbit of 0 under the action of $g$ is $\left(0, \mathrm{III}_{1}, \mathrm{III}_{2}\right)$. There is an edge labeled 3 from $g$ to $s_{i}^{\prime} x^{m} y^{n} \epsilon x^{m} y^{n}=s_{i}^{\prime} x^{2 m} y^{2 n}$.

Consider next $s_{j}$ an instruction of type IX. There are two cases. if $g=s_{j} x y^{n}$ then the orbit of 0 is $\left(0, \mathrm{IX}_{2}, \mathrm{IX}_{4}\right)$ and the output is $\epsilon s_{j}^{\prime} x y^{n} \epsilon$; if $g=s_{j} x^{2 m} y^{n}$ then the orbit of 0 is $\left(0, \mathrm{IX}_{1}, \mathrm{IX}_{4}\right)$ and the output is $\epsilon s_{j}^{\prime \prime} x^{m} y^{n} \epsilon$.

This means that if $m=0$ then there is an edge labeled 3 from $s_{j} x^{2^{m}} y^{2^{n}}$ to $s_{j}^{\prime} x^{2^{m}} y^{2^{n}}$, and if $m>0$ then there is an edge labeled 3 from $s_{j} x^{2^{m}} y^{2^{n}}$ to $s_{j}^{\prime \prime} x^{2^{m-1}} y^{2^{n}}$.

The same naturally applies to instructions of type IX. Finally, for all $m, n \in \mathbb{N}$ the element $s_{\dagger} x^{m} y^{n}$ fixes 0 , and there is an edge labeled 1 from $s_{\dagger} x^{m} y^{n}$ to 1 .

3.4. Contracting automata: proof of Theorem C, We finally explain how to make the transducers $\Phi_{M}$ of the previous subsections contracting. We expand the definition from the introduction:

Definition 3.3 ([16, Definition 2.11.1]). Let $G=\langle\Phi\rangle$ be a self-automata group with $\Phi: A \times S \rightarrow S \times A$ and $\bar{\Phi}: A \times G \rightarrow G \times A$. For $g \in G$ and $u \in A^{*}$, the state $g @ u$ is the unique element of $G$ such that $(u v)^{g}=u^{g} v^{g @ u}$; namely, the action of $g$ on tails of sequences starting with $u$.

The group $G$ is contracting if there exists a finite subset $N \subseteq G$ such that, for all $g \in G$, there exists $n(g) \in \mathbb{N}$ such that $g @ u \in N$ whenever $|u| \geqslant n(g)$.

The minimal subset $N$ satisfying the definition is called the nucleus. In particular, one has $n @ a \in N$ for all $(a, n) \in A \times N$, so $\Phi$ induces an automaton still written $\Phi: A \times N \rightarrow N \times A$. Up to replacing $S$ by $\widetilde{S}:=S \cup N$ and $A$ by $\widetilde{A}:=A^{n}$ for $n$ larger than $\max _{g \in \widetilde{S}^{2}} n(g)$, thus making the transducer process $n$ letters at a time, 
one may also assume

$$
\bar{\Phi}\left(\widetilde{A} \times \widetilde{S}^{2}\right) \subseteq \widetilde{S} \times \widetilde{A} .
$$

A transducer $\Phi$ with this extra property is called nuclear.

Note that is is probably undecidable whether a self-similar group $\langle\Phi\rangle$ is contracting; but it is easy to decide whether a transducer $\Phi: A \times S \rightarrow S \times A$ is nuclear: by minimizing the composite transducer $A \times S^{3} \rightarrow S \times A \times S^{2} \rightarrow S^{2} \times A \times S \rightarrow S^{3} \times A$, find the set $\mathcal{R}$ of all words $s_{1} s_{2} s_{3} \in S^{3}$ that equal 1 in $G$. Then $\Phi$ is nuclear if and only if for all $a \in A, s_{1}, s_{2} \in S$ there exists $s_{3} \in S$ such that if $\Phi\left(a, s_{1}\right)=\left(s_{1}^{\prime}, b\right)$ and $\Phi\left(b, s_{2}\right)=\left(s_{2}^{\prime}, c\right)$ then $s_{1}^{\prime} s_{2}^{\prime} s_{3}^{-1} \in \mathcal{R}$. The more precise form of Theorem $\mathrm{C}$ is:

Theorem 3.4. There is no algorithm that, given a nuclear transducer $\Phi: A \times S \rightarrow$ $S \times A$ and $a \in A$ and $s \in S$, determines the cardinality of the orbit of $a^{\infty}$ under $\langle s\rangle$.

There is no algorithm that, given a nuclear transducer $\Phi: A \times S \rightarrow S \times A$ and $s \in S$, determines the order of $s$ in $\langle\Phi\rangle$.

Note that the group is not changed by these operations of replacing $S$ by $N$ and $A$ by $A^{n}$. If $\Phi$ is nuclear, then $\langle\Phi\rangle$ is contracting in the sense of the introduction, since $\left|g^{\prime}\right| \leqslant(|g|+1) / 2$ in the word metric defined by $N$. Conversely, if $\left|g^{\prime}\right| \leqslant \lambda|g|+C$ then one may take $N=\{g \in G|C /(1-\lambda) \geqslant| g \mid\}$ to see that $G$ is contracting in the sense of Definition 3.3 .

Lemma 3.5. Let $\Phi: A \times S \rightarrow S \times A$ be a transducer. If there is a constant $N \in \mathbb{N}$ such that every reduced path of length $\geqslant N$ in the dual Moore diagram of $\Phi$ contains an $\epsilon$ letter along its output, then $\langle\Phi\rangle$ is contracting.

Proof. Consider $g \in\langle\Phi\rangle$, and represent it by a word $w \in S^{*}$ of length $\ell=|g|$. Factor $w=w_{1} \ldots w_{t}$ with $\left|w_{i}\right|=N$ for all $i=1, \ldots, t-1$ and $\left|w_{t}\right|<N$.

Then every $g^{\prime}$ as in (1.1) is computed by following, in the dual Moore diagram, the path starting at $a_{1}$ with label $w$ on its input. The output label along that path is $g^{\prime}$, and by hypothesis each time a segment $w_{i}$ is read, for $i<t$, an $\epsilon$ letter is produced for $g^{\prime}$; so $\left|g^{\prime}\right| \leqslant \ell-t+1$. Now $t=\lceil\ell / N\rceil$, so

$$
\left|g^{\prime}\right| \leqslant \ell-\lceil\ell / N\rceil+1 \leqslant(1-1 / N)|g|+1 \text {. }
$$

We shall modify the transducers $\Phi_{\mathbf{M}}$ by composing them with appropriate machines. We recall the general definition: let $\Phi: A \times S \rightarrow S \times A$ and $\Psi: B \times S \rightarrow S \times B$ be transducers with same stateset $S$. Their composition is the transducer $\Phi \circ \Psi$ with alphabet $A \times B$, given by

$\Phi \circ \Psi:(A \times B) \times S=A \times(B \times S) \stackrel{A \times \Psi}{\longrightarrow} A \times(S \times B)=(A \times S) \times B \stackrel{\Phi \times B}{\longrightarrow}(S \times A) \times B=S \times(A \times B)$.

We are given a transducer $\Phi$ with stateset $S=\left\{s_{1}, \ldots, s_{\ell}, x, y\right\}$ and alphabet $A$. We write $G=\langle\Phi\rangle$, and freely identify words in $S^{*}$ with their value in $G$. We require that $x, y$ commute.

For every $i \in\{1, \ldots, \ell\}$, consider the transducer $\Phi_{i}$ with alphabet $A_{i}=\{0,1\}$ and transitions $\Phi_{i}(a, q)=\left(a=0 ? q: \epsilon, q=s_{i} ? 1-a: a\right)$.

Note (by drawing the dual Moore diagram and deleting the transitions with $\epsilon$ output) that the only paths with input and output of same length are of the form $s_{i}^{-a} w s_{i}^{b}$ for some $a, b \in\{0,1\}$ and $w$ a word not involving $s_{i}$.

Note also that for a word $w$ of form $s_{j} x^{m} y^{n}$

(1) if $i=j$ then $\Phi_{i}\left(0, w^{2}\right)=(w, 0)$ and $\Phi_{i}\left(1, w^{2}\right)=\left(w^{\prime}, 1\right)$ with $w^{\prime}$ conjugate to $w$ 
(2) if $i \neq j$ then $\Phi_{i}(0, w)=(w, 0)$ and $\Phi_{i}(1, w)=(\epsilon, 1)$.

Consider also a transducer $\Phi_{0}$ with alphabet $A_{0}=\{0,1\}^{3}$ and transitions

$$
\begin{aligned}
& \Phi_{0}\left((a, b, c), s_{i}\right)=\left(c=0 ? s_{i}: \epsilon,(a, b, 1-c)\right) \text { for all } i ; \\
& \Phi_{0}((a, b, c), x)=(a=0 ? x: \epsilon,(1-a, b, c)) ; \\
& \Phi_{0}((a, b, c), y)=(b=0 ? y: \epsilon,(a, 1-b, c)) .
\end{aligned}
$$

Note that, in the dual Moore diagram of $\Phi_{0}$, all paths with input label of the form $s_{i}^{-a} x^{m} y^{n} s_{j}^{b}$ have shorter output label as soon as $|m|+|n| \geqslant 3$. Note also that if $w$ is a word of the form $s_{i} x^{m} y^{n}$ then for all $(a, b, c) \in A_{0}$ we have $\Phi_{0}\left((a, b, c), w^{2}\right)=$ $\left(w^{\prime},(a, b, c)\right)$ for some permutation $w^{\prime}$ of $w$; so in particular $w^{\prime}$ is conjugate to $w$. Furthermore, for $(a, b, c)=(0,0,0)$ we get $w^{\prime}=w$ in $G$.

Proposition 3.6. Under the hypotheses above, the transducer $\Phi^{\prime}:=\Phi \circ \Phi_{0} \circ \Phi_{1} \circ$ $\cdots \circ \Phi_{\ell}$ generates a contracting group, and whenever we have $\Phi\left(a,\left(s_{i} x^{m} y^{n}\right)^{t}\right)=$ $\left(s_{i}^{\prime} x^{m^{\prime}} y^{n^{\prime}}, a\right)$ in the original transducer we have for all $j \in\{0,1\}^{\ell+3}$ the relation $\Phi^{\prime}\left((j, a),\left(s_{i} x^{m} y^{n}\right)^{4 t}\right)=(w,(j, a))$, with $w$ either equal to 1 or conjugate to $s_{i}^{\prime} x^{m^{\prime}} y^{n^{\prime}}$. Furthermore, if $j=0^{\ell+3}$ then $w=s_{i}^{\prime} x^{m^{\prime}} y^{n^{\prime}}$.

Proof. After applying the transducers $\Phi_{1}, \ldots, \Phi_{\ell}$, the only words that don't get shortened are of the form $s_{i}^{-a} w(x, y) s_{j}^{b}$ for some $i, j \in\{1, \ldots, \ell\}$ and some $a, b \in$ $\{0,1\}$. These get shortened by $\Phi_{0}$ as soon as $|w| \geqslant 3$, using the fact that $x$ and $y$ commute. It follows that $\left\langle\Phi^{\prime}\right\rangle$ is contracting.

Consider the transitions of $\left(s_{i} x^{m} y^{n}\right)^{4}$ in transducer $\Phi_{1} \circ \cdots \circ \Phi_{\ell}$. On input letter $0^{\ell}$ it produces $\left(s_{i} x^{m} y^{n}\right)^{2}$, on input letter $0 \cdots 1 \cdots 0$ with the ' 1 ' in position $i$ it produces a conjugate of $\left(s_{i} x^{m} y^{n}\right)^{2}$ and on all other input letters it produces $\epsilon$. Feed then $\left(s_{i} x^{m} y^{n}\right)^{2}$ to transducer $\Phi_{0}$; on input letter 000 it produces $s_{i} x^{m} y^{n}$ and on all other input letters it produces a conjugate of $s_{i} x^{m} y^{n}$. Feed finally $s_{i} x^{m} y^{n}$ to $\Phi$ to conclude the proof.

We are ready to finish the proof of Theorem 3.4. We constructed an integerlabeled graph for a transducer $\Phi$, whose vertices are elements of $G$ for Theorem $\mathrm{B}^{\prime}$ or symmetric conjugacy classes for Theorem B

By Proposition 3.6] the transducer $\Phi^{\prime}$ is contracting. Let us check that the order problems for $\langle\Phi\rangle$ and for $\left\langle\Phi^{\prime}\right\rangle$ are equivalent.

A graph for $\Phi^{\prime}$ will have the same set of vertices as the graph for $\Phi$, and Proposition 3.6 shows that this new graph has the same set of outgoing edges for each element of form $s_{i} x^{m} y^{n}$, with labels multiplied by 4 and, possibly, some new edges to 1 (or to $C(1)$ ). Since in the old graph there were no loops at non-identity elements, $s_{*} x y$ has infinite order in $\left\langle\Phi^{\prime}\right\rangle$ if and only if it has infinite order in $\langle\Phi\rangle$, and the orbit of $\left(0,0^{\ell+3}\right)^{\infty}$ is infinite under the action of $s_{*} x y \in\left\langle\Phi^{\prime}\right\rangle$ if and only if the orbit of $0^{\infty}$ is infinite under the action of $s_{*} x y \in\langle\Phi\rangle$.

Finally, by replacing the stateset $S$ by $\widetilde{S}=S \cup N$ and $A$ by $\widetilde{A}=A^{n}$, we may assume that $\Phi^{\prime}$ is nuclear.

\section{OutLook}

We proved in this article the undecidability of the order problem for automata groups, namely groups of transformations generated by a transducer.

If the transducer belongs to a restricted class, it may well be that the order problem becomes decidable. Of particular importance are: 
Transducers of polynomial growth. In a transducer $\Phi$ (represented by a graph as in Figure 1), let $\alpha(n)$ denote the number of paths of length $n$ that end in a non-identity state. If $\alpha(n)$ is a bounded function (as is the case for the Grigorchuk group), then the order problem is solvable in $\langle\Phi\rangle$, see [3]. What happens if $\alpha(n)$ is bounded by a linear function? or by a polynomial of degree $d$ ? The groups generated by such transducers have been considered by Sidki [17.

Reset transducers. These are transducers $\Phi$ with $\Phi(a, s)=(\phi(a), \psi(a, s))$ for some functions $\phi, \psi$; namely, the state reached by the transducer is independent of the original state. These transducers are intimately connected to tilings, by Kari's construction [12]. Gillibert proved in [5] that the order problem is unsolvable for semigroups of reset automata. Is it solvable in groups of reset automata?

Reversible transducers. These are transducers whose dual is invertible; they should be related to reversible Turing or Minsky machines. Is the order problem solvable for groups generated by reversible automata?

Bireversible transducers. These are transducers $\Phi$ such that all 8 transducers obtained from $\Phi$ by inverting or permuting the stateset and alphabet remain transducers; they give another point of view on square complexes (by tiling the plane with squares whose labels are $\left(a, s, a^{\prime}, s^{\prime}\right)$ when $\left.\Phi(a, s)=\left(s^{\prime}, a^{\prime}\right)\right)$.

We expect it to be undecidable whether a functionally recursive group is actually an automata group (for a larger generating set), whether an automata group is contracting, and even whether a contracting group is finite. Again, the related questions for semigroups are known to be undecidable by constructions in or similar to $[5]$.

\section{REFERENCES}

[1] Ali Akhavi, Ines Klimann, Sylvain Lombardy, Jean Mairesse, and Matthieu Picantin, On the finiteness problem for automaton (semi)groups, Internat. J. Algebra Comput. 22 (2012), no. 6, 1250052, 26, DOI 10.1142/S021819671250052X. MR2974106

[2] Robert Berger, The undecidability of the domino problem, Mem. Amer. Math. Soc. No. 66 (1966), 72. MR0216954 (36 \#49)

[3] Ievgen V. Bondarenko, Natalia V. Bondarenko, Said N. Sidki, and Flavia R. Zapata, On the conjugacy problem for finite-state automorphisms of regular rooted trees, Groups Geom. Dyn. 7 (2013), no. 2, 323-355, DOI 10.4171/GGD/184. With an appendix by Raphaël M. Jungers. MR3054572

[4] Andrew M. Brunner and Said N. Sidki, On the automorphism group of the one-rooted binary tree, J. Algebra 195 (1997), no. 2, 465-486. MR98i:20035

[5] Pierre Gillibert, The finiteness problem for automaton semigroups is undecidable, Internat. J. Algebra Comput. 24 (2014), no. 1, 1-9, DOI 10.1142/S0218196714500015. MR3189662

[6] _ An automaton group with undecidable order and Engel problems (2017), available at $\operatorname{arXiv:1710.09733}$

[7] Rostislav I. Grigorchuk, On Burnside's problem on periodic groups, Функционал. Анал. и Приложен. 14 (1980), no. 1, 53-54. English translation: Functional Anal. Appl. 14 (1980), 41-43. MR81m:20045

[8] - On the Milnor problem of group growth, Dokl. Akad. Nauk SSSR 271 (1983), no. 1, 30-33. MR85g:20042

[9] Rostislav I. Grigorchuk, Volodymyr V. Nekrashevych, and Vitaliı I I Suščans'kiǔ, Automata, dynamical sysytems, and groups, PROSI 231 (2000), no. 4, 128-203.

[10] Narain D. Gupta and Said N. Sidki, On the Burnside problem for periodic groups, Math. Z. 182 (1983), 385-388.

[11] Ju. Š. Gurevič, The problem of equality of words for certain classes of semigroups, Algebra i Logika Sem. 5 (1966), no. 5, 25-35 (Russian). MR0206079 
THE WORD AND ORDER PROBLEMS FOR SELF-SIMILAR AND AUTOMATA GROUPS 17

[12] Jarkko Kari, The nilpotency problem of one-dimensional cellular automata, SIAM J. Comput. 21 (1992), no. 3, 571-586, DOI 10.1137/0221036. MR1163346

[13] Ines Klimann, Jean Mairesse, and Matthieu Picantin, Implementing computations in automaton (semi)groups, Implementation and application of automata, Lecture Notes in Comput. Sci., vol. 7381, Springer, Heidelberg, 2012, pp. 240-252, DOI 10.1007/978-3-642-31606-7_21. MR2993189

[14] Ville Lukkarila, The 4-way deterministic tiling problem is undecidable, Theoret. Comput. Sci. 410 (2009), no. 16, 1516-1533, DOI 10.1016/j.tcs.2008.12.006. MR2502125 (2010d:03071)

[15] Marvin L. Minsky, Recursive unsolvability of Post's problem of "tag" and other topics in theory of Turing machines, Ann. of Math. (2) 74 (1961), 437-455, DOI 10.2307/1970290. MR0140405

[16] Volodymyr V. Nekrashevych, Self-similar groups, Mathematical Surveys and Monographs, vol. 117, American Mathematical Society, Providence, RI, 2005. MR2162164 (2006e:20047)

[17] Said N. Sidki, Automorphisms of one-rooted trees: growth, circuit structure, and acyclicity, J. Math. Sci. (New York) 100 (2000), no. 1, 1925-1943. Algebra, 12. MR1774 362

Département de Mathématiques et Applications, École Normale Supérieure, Paris and Mathematisches Institut, Georg-August Universität zu Göttingen

E-mail address: laurent.bartholdi@gmail.com

E-mail address: phortim@yandex.ru 